\title{
Norois
}

Environnement, aménagement, société

$251 \mid 2019$

L'expérience de la catastrophe

\section{Postface : enjeux et défis du programme ATLANTYS}

Afterword: Issues and Challenges of the Atlantys Program

\section{Frédéric Le Blay}

\section{(2) OpenEdition}

Journals

Édition électronique

URL : https://journals.openedition.org/norois/9246

DOI : $10.4000 /$ norois. 9246

ISSN : 1760-8546

\section{Éditeur}

Presses universitaires de Rennes

\section{Édition imprimée}

Date de publication : 22 novembre 2019

Pagination : 115-117

ISBN : 978-2-7535-7875-3

ISSN : 0029-182X

\section{Référence électronique}

Frédéric Le Blay, "Postface : enjeux et défis du programme ATLANTYS », Norois [En ligne], 251 | 2019, mis en ligne le 21 novembre 2019, consulté le 08 janvier 2022. URL : http://journals.openedition.org/ norois/9246 ; DOI : https://doi.org/10.4000/norois.9246 


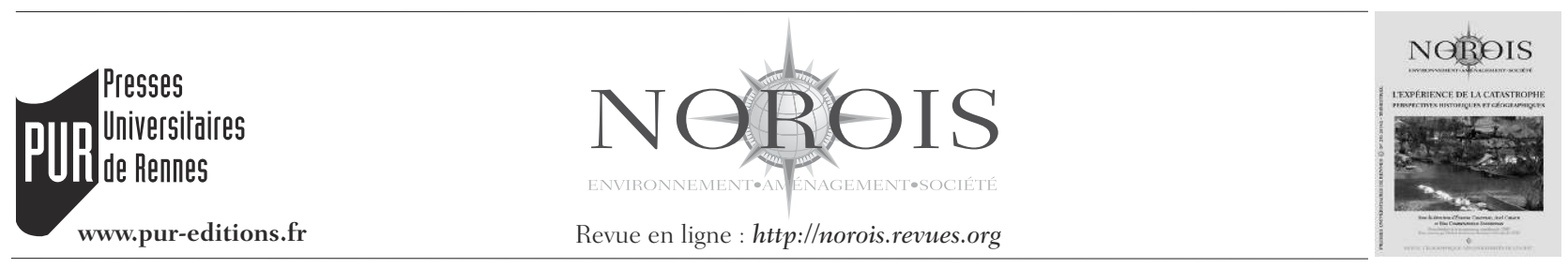

\title{
Postface : enjeux et défis du programme ATLANTYS
}

\author{
Frédéric Le Blay
}

Université de Nantes, Centre François-Viète (EA 1161), Coordinateur scientifique d'ATLANTYS. (frederic.le-blay@

univ-nantes.fr)

\section{Suivie d'un témoignage d'expert par Frederick SPIELBERG (Unicef-Colombie)}

\section{L'INTERDISCIPLINARITÉ ET SES ENJEUX DU POINT DE VUE D'ATLANTYS : RÉFLEXIONS CONCLUSIVES}

La thématique du colloque dont sont issus les articles publiés dans ce numéro de la revue Norois présentait assez nettement une double orientation historique et géographique - reflet de l'orientation générale de l'axe du programme Atlantys qui le portait, mais cette rencontre fut aussi et surtout l'occasion de pratiquer une forme d'interdisciplinarité au sens fort. L'interdisciplinarité est cependant devenue une bannière que l'on agite de manière systématique dans un contexte où l'institution académique encourage à faire éclater les frontières disciplinaires pour justifier de sa capacité à répondre aux grands enjeux sociétaux du temps. Et même si l'on est acquis aux vertus de l'approche interdisciplinaire, il n'en est pas moins bienvenu de se poser la question de sa pertinence et de sa définition. Sommes-nous confrontés à un effet de mode qui passera dès qu'il aura atteint ses limites ou bien s'agit-il d'une évolution profonde et durable de nos conceptions et de nos pratiques académiques et scientifiques? Deux postures, l'une historique, l'autre épistémologique, peuvent être invoquées pour tenter de répondre à ce questionnement.

Premièrement, la séparation du domaine de la connaissance et de la spéculation théorique en disciplines aux frontières et objets strictement définis est une évolution récente dans l'histoire de la pensée. Elle est concomitante de la constitution au cours de la seconde moitié du XIX siècle, en Europe et en Amérique, de l'Université moderne et des grandes institutions de recherche publiques. Il fallait alors organiser la science et le savoir en grands domaines, eux-mêmes subdivisés en sous-domaines, susceptibles de baliser les champs de compétence et d'expertise des uns et des autres, autant en vue d'assurer leur reconnaissance que d'asseoir et garantir l'autorité des «maîtres » sur leurs territoires intellectuels respectifs ${ }^{1}$. L'émergence de disciplines nouvelles

1. Cette tendance académique est très bien résumée par Nathan Sivin (2018), Why some comparisons make more difference than others, in Lloyd G.E.R., Zhaо J.J. (ed.), Ancient Greece and China Compared, Cambridge University Press, p. 33-39. De manière générale, je renvoie aux trois premiers textes méthodologiques du volume collectif présentant cette contribution, qui posent les termes et les conditions d'une approche comparatiste de l'histoire (ici entre les civilisations de l'Antiquité classique 
(linguistique, anthropologie, ethnologie, sociologie, etc.) qui recherchèrent d'emblée la reconnaissance institutionnelle qui leur était légitimement due n'a sans doute fait que renforcer ce mouvement de parcellisation de la recherche universitaire, chacun devant pouvoir, afin d'exister, s'inscrire dans un paysage constitué de balises et d'objets dûment identifiés et labellisés. Cette lecture historique se heurte naturellement à des contre-exemples locaux et il conviendrait d'affiner le propos pour ne pas tomber dans le schématisme caricatural. Si l'on se tourne néanmoins vers les périodes antérieures, en se gardant bien entendu de toute nostalgie déplacée, on constate la grande souplesse intellectuelle des savants et des hommes de science. Les grands textes théoriques de l'Antiquité sont le fait le plus souvent de polygraphes qui conçoivent la spéculation tantôt sur le mode de la curiosité tantôt sur le mode de l'entreprise philosophique, selon une conception globalisante héritée de l'aristotélisme. Cette manière de faire la science perdure bien au-delà de l'Antiquité. C'est l'une des dimensions que nous avons souhaité mettre en avant dans un travail de mise en perspective de la pensée de Descartes au regard de la tradition aristotélicienne, dont la conclusion était la suivante :

"L'histoire des sciences et de la pensée européennes, dont on veut - avec une incontestable justesse - qu'elle commence en Grèce et à Rome, est ainsi semée de penseurs ou de courants philosophiques qui posent l'encyclopédisme, au sens propre (le cercle des connaissances), comme voie d'accès à la sagesse. Cette histoire commence avec Aristote; elle connaît ensuite des renaissances étalées dans le temps, le XIII ${ }^{\mathrm{e}}$ siècle, l'Humanisme, les Lumières. Cette posture systématique est peut-être le moteur et l'enjeu de la modernité. Descartes appartient à cette histoire. En ce sens, si l'on dit de Descartes qu'il incarne le génie français, il convient surtout de voir en lui un représentant de la pensée européenne appartenant autant à l'histoire des sciences qu'à celle de la philosophie. Et face à ce modèle et en guise de conclusion, nous proposerons très simplement d'interroger la modernité de notre époque ${ }^{2}$. »

et les anciennes civilisations de l'Asie orientale) et de la notion de Global History, récemment introduite dans le paysage académique.

2. Le Blay Frédéric (2012), Descartes contre Aristote : l'autre querelle des Anciens et des Modernes, in Jullien V., Nicolaidis E., avec Blay M.
Deuxièmement, il semble que cette interdisciplinarité, si on veut continuer à l'appeler ainsi, soit l'apprentissage de la pensée complexe. Nous reprenons ici volontiers cette notion défendue par Edgar Morin dans un essai qui fit date, Science avec conscience ${ }^{3}$. Non seulement les questions que nous abordons dans le cadre du programme ATLANTYS sont si fondamentales qu'elles échappent à la classification et à l'enfermement disciplinaires mais elles sont aussi si pressantes qu'il est difficile de ne les aborder que par le seul biais de la spéculation théorique. D'une manière ou d'une autre, de telles questions engagent celui qui les pose dans la complexité du réel et donc en dehors des seuls murs de l'institution académique. Il est évident que le beau rêve humaniste de l'homme de tous les savoirs est une utopie que cette même complexité du réel rend pratiquement caduque à l'échelle d'une vie. De ce point de vue, nous avons besoin de notre ancrage disciplinaire pour pouvoir développer une quelconque expertise. Mais la mise en pratique de la réflexion collective et partagée autour d'objets complexes qui ne soient pas marqués disciplinairement est une démarche nécessaire. Cela n'a rien d'une évidence car du fait de la formation que nous recevons, nous sommes profondément marqués par des méthodes et des manières de penser liées à nos choix disciplinaires; aussi parce que, dans le vaste domaine des Lettres et des Sciences humaines en particulier, la culture du chercheur a longtemps été celle du travail solitaire dans la quiétude bienveillante et rassurante de sa bibliothèque ou de son cabinet. Cette quiétude offre le cadre propice à la réflexion mais elle peut aussi conduire à l'enfermement et à une pensée autocentrée.

\section{La démonstration par les faits}

Forts de ces principes, il nous devenait impératif non seulement de faire bouger les lignes entre disciplines scientifiques et académiques mais également de ne pas enfermer le propos et la démarche dans le cercle restreint de la communauté scientifique. C'est pourquoi, à l'occasion de nos différentes rencontres internationales - appelons-les « colloques » confor-

(éd.), Europe et sciences modernes. Histoire d'un engendrement mutuel, coll. «Dynamiques citoyennes en Europe », 2, Peter Lang, Bern/Berlin/ Bruxelles/Frankfurt am Main-New York/Oxford/Wien, p. 231-246.

3. Morin Edgar (1982), Science avec conscience, Fayard, Paris (Éditions du Seuil, Coll. «Points/Sciences »). 
mément à l'usage établi, nous avons tenu à nous adresser aussi au grand public, en invitant certains de nos collègues à ne pas proposer une communication sous la forme "scientifique " généralement requise mais plutôt une conférence faisant état d'un parcours intellectuel et d'une réflexion personnelle, le tout néanmoins soutenu par l'expertise scientifique; c'est ici le cas du point de vue exposé par Emanuela Guidoboni, que nous avons retenu pour ce numéro thématique. Mais nous avons aussi fait intervenir des experts de «terrain » capables de dialoguer avec nous en nous faisant bénéficier de leur expérience et de leur regard. Frederick Spielberg fut de ces intervenants : anthropologue de formation et spécialisé en politiques publiques, il a travaillé depuis 25 ans dans le secteur humanitaire pour les
Nations Unies et des ONGs, dans plus de 80 pays. Entre 2005 et 2013, il a été responsable de la préparation aux situations d'urgences à l'échelle mondiale pour l'Unicef. Actuellement il est responsable de la section « urgence migratoire » au bureau de l'Unicef de Bogota en Colombie. Depuis son bureau de Bogota, il a bien voulu s'adresser à nous et au public dans un entretien sous forme d'exposé synthétique, suivi d'échanges. Nous sommes heureux de pouvoir présenter ici, en guise de conclusion aux travaux réunis, une version remaniée et abrégée de son exposé liminaire. Plusieurs des aspects évoqués (gestion du risque, résilience, réduction des désastres, responsabilité) étaient en effet au cœur des échanges tenus lors du colloque de novembre 2016. 